\title{
(Ir)responsabilidade social empresarial: uma avaliação do desastre de Mariana-MG
}

Mirian Albert Pires 1

Luciana Mourão ${ }^{2}$

Fátima Bayma de Oliveira ${ }^{3}$

Jose Antonio Puppim de Oliveira ${ }^{34}$

1 Universidade Federal do Espírito Santo / Departamento de Ciências Contábeis, Vitória / ES - Brasil

2 Universidade Salgado de Oliveira / Programa de Pós-Graduação em Psicologia, Niterói / RJ - Brasil

${ }^{3}$ Fundação Getulio Vargas / Escola Brasileira de Administração Pública e de Empresas, Rio de Janeiro / RJ - Brasil

${ }^{4}$ Fundação Getulio Vargas / Escola de Administração de Empresas de São Paulo, São Paulo / SP - Brasil

A temática irresponsabilidade social empresarial (IrSE) ganhou destaque na literatura mundial. No Brasil, o desastre causado pela Samarco Mineração S.A. em 2015 foi alvo de repercussão, constituindo uma oportunidade para estudar a IrSE. O objetivo deste estudo foi investigar se a identificação social com a empresa, que surge em função dos benefícios econômicos, reduz a intenção punitiva e a atribuição de culpa. O instrumento de pesquisa contém escalas com indicadores psicométricos aplicados a 1.616 indivíduos. Constatou-se que os benefícios para a economia local reduziram a intenção punitiva nas cidades de Anchieta-ES, Guarapari-ES e Mariana-MG. O que não ocorreu nas cidades de Colatina-ES e Linhares-ES, que não recebem benefícios econômicos, mas foram afetadas pelo desastre. Identificou-se, ainda, que a severidade do evento aumentou a atribuição de culpa e a intenção punitiva aumentou a intenção de comentários negativos. Como principal conclusão, onde a empresa gerou benefícios, as pessoas estavam menos propensas a puni-la.

Palavras-chave: irresponsabilidade social empresarial; benefícios econômicos; atribuição de culpa; intenção punitiva; desastre ambiental.

\section{(Ir)responsabilidad social empresarial: evaluación del desastre de Mariana-MG}

La temática irresponsabilidad social empresarial (IrSE) ganó prominencia en la literatura mundial. En Brasil, el desastre causado por Samarco Mineração S.A. en 2015 fue objeto de repercusión, constituyendo una oportunidad para estudiar la IrSE. El objetivo de este estudio fue investigar si la identificación social con la empresa, que surge en función de los beneficios económicos, reduce la intención punitiva y la atribución de culpa. El instrumento de investigación contiene escalas con indicadores psicométricos aplicados a 1.616 individuos. Se constató que los beneficios para la economía local redujeron la intención punitiva en las ciudades de Anchieta, ES; Guarapari, ES y Mariana, MG. Lo que no ocurrió en las ciudades de Colatina, ES y Linhares-ES, que no reciben beneficios económicos y fueron afectadas por el desastre. Se identificó además que la severidad del acontecimiento aumentó la atribución de culpa y la intención punitiva aumentó la intención de comentarios negativos. Como conclusión, donde la empresa generó beneficios, la gente estaba menos propensa a castigarla.

Palabras clave: irresponsabilidad social empresarial; beneficios económicos; atribución de culpa; intención punitiva; desastre ambiental.

\section{Corporate social (ir)responsibility: an analysis of the disaster in Mariana-Brazil}

The issue of corporate social irresponsibility (CSIR) has gained prominence in world literature. This study analyzes the 2015 environmental disaster caused by Samarco Mineração S.A. in Mariana (MG), Brazil, as an example of CSIR. The objective was to understand whether the population's social identification with the company, which is translated into the economic benefits, reduced punitive intention, and blame attribution. The research instrument uses scales with psychometric indicators applied to 1,616 individuals. It was verified that the benefits to the local economy reduced the punitive intention in the municipalities of Anchieta and Guarapari (ES), and Mariana (MG). This was not the case for Colatina and Linhares (ES). Also, it was observed that the disaster's severity increased blame attribution, and the punitive intention increased the intention to engage in negative word-of-mouth (NWOM) about the company. As the main conclusion, people were less likely to punish the company in the municipalities where it generated economic benefits. Keywords: corporate social irresponsibility, economic benefits, blame attribution, punitive intention, environmental disaster. 


\section{AGRADECIMENTOS}

Os autores agradecem à Coordenação de Aperfeiçoamento de Pessoal de Nível Superior (CAPES).

\section{INTRODUÇÃO}

A irresponsabilidade social empresarial (IrSE) é uma vertente ligada à temática responsabilidade social empresarial (RSE), que vem chamando atenção (Alexander, 2015; Kotchen \& Moon, 2012; Popa \& Salanță, 2014). Todavia, a IrSE nem sempre foi discutida de maneira explícita na literatura sobre a RSE (Lange \& Washburn, 2012), sugerindo a necessidade de aprofundamento e desenvolvimento de pesquisas.

Entre as definições, destaca-se a de Armstrong (1977, p. 185, tradução nossa), ao apresentar que "um ato socialmente irresponsável [...] envolve um ganho para uma das partes em detrimento de todo o sistema". Alguns consideram que essa foi a primeira definição sobre a temática (Murphy \& Schlegelmilch, 2013; Olofsson \& Söderholm, 2014). A título de exemplos de IrSE, apontam-se: fixar preços, violar direitos humanos, danos ao meio ambiente, prestar informações imprecisas sobre produtos e enganar os clientes (Lin-Hi \& Müller, 2013).

Contudo, para que a sociedade classifique um ato praticado por uma empresa como socialmente responsável ou irresponsável, esse julgamento leva em consideração um conjunto de dimensões culturais (Lange \& Washburn, 2012). Além disso, países em desenvolvimento geralmente têm dificuldade de dar atenção à responsabilidade social, pois as pessoas têm outras necessidades primárias, como seu próprio sustento e o de suas famílias (Martínez-Alier, 1995). Nesses países com maior precariedade social, é comum encontrar maior dependência dos benefícios econômicos que as empresas trazem para a localidade (Velasquez, 2012), o que desestimula a sociedade a agir contra elas quando incorrem em atos de IrSE (Bazerman \& Tenbrunsel, 2011).

Esses apontamentos trazem à tona a realidade do Brasil. Nesse contexto, a pesquisa se propôs a analisar como um ato de IrSE é percebido pelos cidadãos de uma localidade, a partir do caso do rompimento da barragem de Fundão, no distrito de Bento Rodrigues, na cidade de Mariana-MG, ocorrido em novembro de 2015, em que 40 municípios foram impactados pelo desastre. $\mathrm{O}$ foco da pesquisa se voltou a cinco municípios que tiveram impactos diferentes, quais sejam: Anchieta-ES, Colatina-ES, Guarapari-ES, Linhares-ES e Mariana-MG.

Apresenta-se, ainda, que a investigação teve como foco identificar sentimentos e julgamentos que surgiram a partir da forma como esses indivíduos perceberam o referido ato. Complementando, destaca-se que a Samarco Mineração S.A., empresa causadora desse evento, é controlada pela companhia brasileira Vale S.A. e pela anglo-australiana BHP Billiton (Samarco Mineração S.A., 2016). Assim, constrói-se a seguinte questão:

- A existência de identificação social com a empresa, que se dá em função dos benefícios econômicos proporcionados por ela a algumas cidades, reduz a intenção punitiva a um evento de IrSE e a atribuição de culpa dessa empresa?

Essa questão possibilitou o seguinte objetivo:

- Investigar se a identificação social com a empresa, que surge em função dos benefícios econômicos por ela proporcionados a alguns municípios, reduz a intenção punitiva e a atribuição de culpa, usando o caso da Samarco Mineração S.A. no contexto do acidente da barragem do Fundão. 
Esta pesquisa traz um conjunto de contribuições para a literatura nacional e internacional, dentre as quais destacamos cinco. Primeiro, traz à tona o conceito de IrSE no contexto brasileiro. Considera-se relevante avaliar o comportamento de indivíduos do Brasil quando se deparam com um ato de IrSE, tendo em vista que a temática ainda não foi muito explorada no cenário nacional. A relevância do estudo fica ainda maior em localidades nas quais a população demonstrou uma identificação social com a empresa causadora do desastre, em virtude dos benefícios econômicos proporcionados. Segundo, proporciona reflexão sobre o papel do benefício econômico na formação da identificação social da empresa e sua influência sobre a atitude dos indivíduos. Terceiro, discute como indivíduos afetados de formas diferenciadas por um ato de IrSE o percebem e o avaliam, dado que são escassos estudos empíricos na área. Quarto, os achados evidenciam que, dado o benefício econômico da empresa, os indivíduos são menos propensos a puni-la, o que pode ter desestimulado a controladora Vale S.A. a tomar as medidas cabíveis, visando a evitar outros desastres, como o ocorrido em janeiro de 2019 em Brumadinho-MG, causando danos ambientais, materiais e a morte de centenas de pessoas (O Portal de Notícias da Globo, 2019a). Quinto, em relação ao cenário internacional, considerando que os achados se referem a um país em desenvolvimento, torna-se possível compará-los com aqueles oriundos de países com outra condição socioeconômica e cultural.

\section{REFERENCIAL TEÓRICO E HIPÓTESES}

Ao longo da história, o tema IrSE despertou o interesse para o desenvolvimento de pesquisas, apontando-se trabalhos variados, como aqueles que abordam a disposição dos consumidores em punir empresas que praticam IrSE (Sweetin, Knowles, Summey, \& McQueen, 2013), que relacionam a IrSE com o desempenho da firma (Kang, Germann, \& Grewal, 2016; Walker, Zhang, \& Ni, 2019), a inclinação de consumidores a realizar boicotes, protestos e comentários negativos às empresas irresponsáveis (Trautwein \& Lindenmeier, 2019), a permanência e a sucessão dos executivos (Chiu \& Sharfman, 2018; Oh, Chang, \& Jung, 2018) e o preço das ações (Lee, Lu, \& Wang, 2019).

Não obstante, também se infere que sempre existiram casos de empresas que foram ou ainda são "ambientalmente danosas", que venderam produtos prejudiciais à saúde, que exploraram funcionários ou que colaboraram para o aquecimento global. Esses eventos ocorrem, em sua maioria, em países subdesenvolvidos ou em desenvolvimento. Todavia, a população e os governos desses países raramente punem tais empresas para evitar danos futuros. A principal razão provavelmente se associa aos benefícios econômicos dessas empresas, como o aumento de empregos, o estímulo à economia, além da contribuição ao produto interno bruto (PIB) (Bazerman \& Tenbrunsel, 2011; George, Kuye, \& Onokala, 2012; Hilson, 2012).

Contudo, essas situações também ocorrem em países desenvolvidos, como nos Estados Unidos da América (EUA), onde existiu a exposição de funcionários ao amianto e a venda de automóveis com problemas de fabricação (Schwartz \& Carroll, 2003; Schwarze, 2003). Essa realidade aponta um conflito de moralidade, dado que, mesmo quando a empresa provoca malefícios, a população não almeja que encerre suas atividades, pois a economia é impulsionada por ela. Desse modo, a fim de trazer explicações, existe o entendimento de que uma reivindicação social justificada aos olhos de um grupo de interesse social pode apresentar-se de forma diferente das ideias morais dos gerentes, fornecedores, clientes ou outros grupos (Scherer \& Palazzo, 2007). Esse cenário, no qual diferentes grupos sociais se manifestam, relaciona-se com a teoria da identificação social, que procura escrever, 
prescrever e avaliar os fatos, gerando uma opinião consensual e compartilhada pelo grupo e buscando um favorecimento interno (Hogg, Terry, \& White, 1995).

Para além da teoria da identificação social, também é preciso considerar a teoria da atribuição, que fundamenta o ato de imputar culpa ao causador de um evento danoso. Tal entendimento toma por base a analogia de Hamilton (1980), que coloca o observador tanto como um psicólogo intuitivo, visando a julgar a causalidade, quanto um advogado intuitivo, julgando sua responsabilidade moral. Todavia, existem vieses de julgamento da atribuição de culpa. Assim, muitos desastres que foram causados por empresas e trouxeram consequências negativas foram percebidos de forma diferente pelos seus observadores devido a fatores morais, éticos e culturais (Lange \& Washburn, 2012).

No caso da Samarco Mineração S.A., por exemplo, a Prefeitura de Anchieta defendeu a continuidade de seu funcionamento, apresentando os efeitos negativos que a paralisação da mineradora trouxe, já que ela era a maior responsável, de forma direta e indireta, pelo pagamento de tributos (Prefeitura Municipal de Anchieta, 2017). Por sua vez, as prefeituras de Guarapari e Mariana também destacaram a importância econômica da mineradora (Augusto, 2016; Avila, 2015). Todos esses apontamentos contribuíram para aumentar a identificação social com a firma e afetaram a forma como os indivíduos analisam a realidade, conforme previsto na perspectiva teórica da sociologia do conhecimento (Berger \& Luckmann, 1985). Diante do exposto, é possível formular a seguinte hipótese:

Hipótese 1: A percepção dos moradores de Anchieta-ES, Colatina-ES, Guarapari-ES, Linhares-ES e Mariana-MG acerca de benefícios proporcionados pela Samarco Mineração S.A. para a economia local afeta negativamente a atribuição de culpa à empresa por parte de tal público em relação ao desastre causado pela empresa em 2015.

A hipótese seguinte se propõe a testar a relação teórica entre a atribuição de culpa e a intenção punitiva em um contexto de desastre ocorrido no Brasil. Um dos pontos relevantes para essa discussão se refere ao fato de que quando o observador tem a crença de que a responsabilidade moral da empresa é baixa, as avaliações de culpa tendem a ser menores, mesmo que existam percepções altas de causalidade (Lange \& Washburn, 2012). Assim, diferentes elementos podem influenciar os observadores, o que contribui para explicar avaliações distintas.

Entretanto, essas diferenças de percepções individuais não alteram o fato da intenção punitiva se conectar com a atribuição de culpa ou a responsabilidade por algum evento (Olofsson \& Söderholm, 2014), uma vez que existe relação entre a teoria da equidade e a da atribuição. Desse modo, tais argumentações sustentam esta hipótese:

Hipótese 2: A atribuição de culpa por parte dos moradores de Anchieta-ES, Colatina-ES, Guarapari-ES, Linhares-ES e Mariana-MG acerca do desastre causado pela Samarco Mineração S.A. em 2015 afeta positivamente a intenção de punir a empresa.

Nesse contexto, a atribuição de culpa também teria outro fator impactante, sendo a severidade ou a gravidade percebida (Kelley \& Michela, 1980). Isso reforça a ideia de diferenças individuais na 
percepção dos danos causados pelas empresas, dado que as emoções emanadas nem sempre são iguais. Portanto, a depender da percepção da severidade, os julgamentos de atribuição de culpa serão mais ou menos rígidos (Antonetti \& Maklan, 2016a; Kelley \& Michela, 1980).

Lange e Washburn (2012) alertam para duas características que irão chamar atenção quanto ao dano, quais sejam: a inexpressividade e a concentração no tempo e no espaço. Assim, eventos com alta expressividade e concentração geralmente são classificados como severos. Tal entendimento leva em consideração que fatores salientes influenciam a atribuição causal (McArthur \& Ginsberg, 1981), o que possibilita o desenvolvimento desta hipótese:

Hipótese 3: A avaliação de severidade ou gravidade por parte dos moradores de Anchieta-ES, Colatina-ES, Guarapari-ES, Linhares-ES e Mariana-MG relativamente ao desastre causado pela Samarco Mineração S.A. em 2015 afeta positivamente a atribuição de culpa da empresa por parte de tal público.

Outro ponto a ser discutido é o fato da identificação social influenciar as percepções de injustiça. Esse entendimento encontra respaldo no trabalho de Campbell (1995), que evidenciou a existência de várias correntes de pesquisa ligadas à teoria da equidade, tratando das percepções de equidade, justiça distributiva e preços justos. De acordo com o autor, as percepções de injustiça se baseiam na comparação entre grupos, portanto, a percepção de injustiça varia entre pessoas. Inclusive, o desastre ambiental ocasionado pela mineradora causou sua paralisação, gerando desemprego e queda na movimentação financeira, o que pode afetar a intenção punitiva. Tais apontamentos possibilitam a construção desta hipótese:

Hipótese 4: A percepção dos moradores de Anchieta-ES, Colatina-ES, Guarapari-ES, Linhares-ES e Mariana-MG acerca de benefícios proporcionados pela Samarco Mineração S.A. para a economia local afeta negativamente a intenção punitiva de tal público em relação ao desastre causado pela empresa em 2015.

Outro ponto presente nas discussões de IrSE é a relação entre severidade/gravidade e a intenção punitiva. Dunford e Ridley (1996) demonstraram que quanto maior o dano, maior a intensidade da punição. Nordgren e McDonnell (2010) também apresentaram a relação teórica entre a severidade de um evento e a punição aplicada. Por sua vez, Carlsmith, Darley, e Robinson (2002) comentaram que existe a expectativa de que o infrator seja punido na mesma proporção da severidade do dano. Cabe apontar que tanto a severidade quanto a atribuição de culpa apresentam vínculo com a teoria da atribuição e que a intenção punitiva guarda relação com a teoria da equidade. Sendo assim, torna-se possível a construção desta hipótese:

Hipótese 5: A avaliação de severidade ou gravidade por parte dos moradores de Anchieta-ES, Colatina-ES, Guarapari-ES, Linhares-ES e Mariana-MG relativa ao desastre causado pela Samarco Mineração S.A. em 2015 afeta positivamente a intenção de punir a empresa.

Explorando os elementos que antecedem a intenção de realizar comentários negativos se encontra a atribuição de culpa. Esse entendimento toma como discussão o trabalho de Ping, Ishaq, e Li (2015), 
que fez uma análise do processo de atribuição de culpa e evidenciou que ela influencia reações negativas dos consumidores. Exemplo disso são estudos apontando evidências de que a atribuição de culpa afeta positivamente os comentários negativos (Richins, 1983, 1987), em consonância com a teoria da atribuição. Desse modo, constrói-se a seguinte hipótese:

Hipótese 6: A avaliação de atribuição de culpa da empresa por parte dos moradores de Anchieta-ES, Colatina-ES, Guarapari-ES, Linhares-ES e Mariana-MG acerca do desastre causado pela Samarco Mineração S.A. em 2015 afeta positivamente a intenção de fazer comentários negativos sobre a empresa.

Ainda em relação à intenção de fazer comentários negativos, um fato primário, cuja existência se mostra necessária, é a percepção de severidade do evento. Tal necessidade foi retratada pela literatura, apontando que quanto maior a atribuição de severidade a um evento, maior a intenção da sociedade de fazer comentários negativos (Antonetti \& Maklan, 2016a). Essa visão também foi defendida por Chang, Tsai, Wong, Wang e Cho (2015), ao argumentarem que a severidade da falha ocasionava a reação de comentários negativos. Essas argumentações encontram suporte na teoria da atribuição, tendo em vista que a atribuição de severidade afeta a intenção de fazer comentários negativos. Sob essa lógica se construiu a seguinte hipótese:

Hipótese 7: A avaliação de severidade ou gravidade do evento por parte dos moradores de Anchieta-ES, Colatina-ES, Guarapari-ES, Linhares-ES e Mariana-MG acerca do desastre causado pela Samarco Mineração S.A. em 2015 afeta positivamente a intenção de fazer comentários negativos sobre a empresa.

Ainda como uma das variáveis que antecedem os comentários negativos, é possível apresentar a intenção punitiva. Essa relação se dá em função desses comentários atuarem como resposta da sociedade, representando uma forma de punição. Inclusive, Buttle (1998) menciona três ações punitivas adotadas pela sociedade contra a empresa: sair do relacionamento, demonstrar insatisfação e fazer comentários negativos. A teoria que sustenta essa relação é a teoria da equidade, dado que a intenção de fazer comentários negativos surgiria em função da iniquidade praticada. Ressalta-se ainda que, em situações de raiva, as pessoas despertam o interesse em realizar punições, e uma dessas formas seria falar mal para outros. Posto isso, constrói-se esta hipótese:

Hipótese 8: A intenção punitiva por parte dos moradores de Anchieta-ES, Colatina-ES, Guarapari-ES, Linhares-ES e Mariana-MG acerca do desastre causado pela Samarco Mineração S.A. em 2015 afeta positivamente a intenção de fazer comentários negativos sobre a empresa.

Tomadas em conjunto, essas hipóteses permitem a construção do modelo relacionando os benefícios econômicos e a IrSE, apresentado na Figura 1. Tal modelo é suportado pelas teorias apresentadas e um conjunto de achados empíricos citados nesta revisão de literatura. 


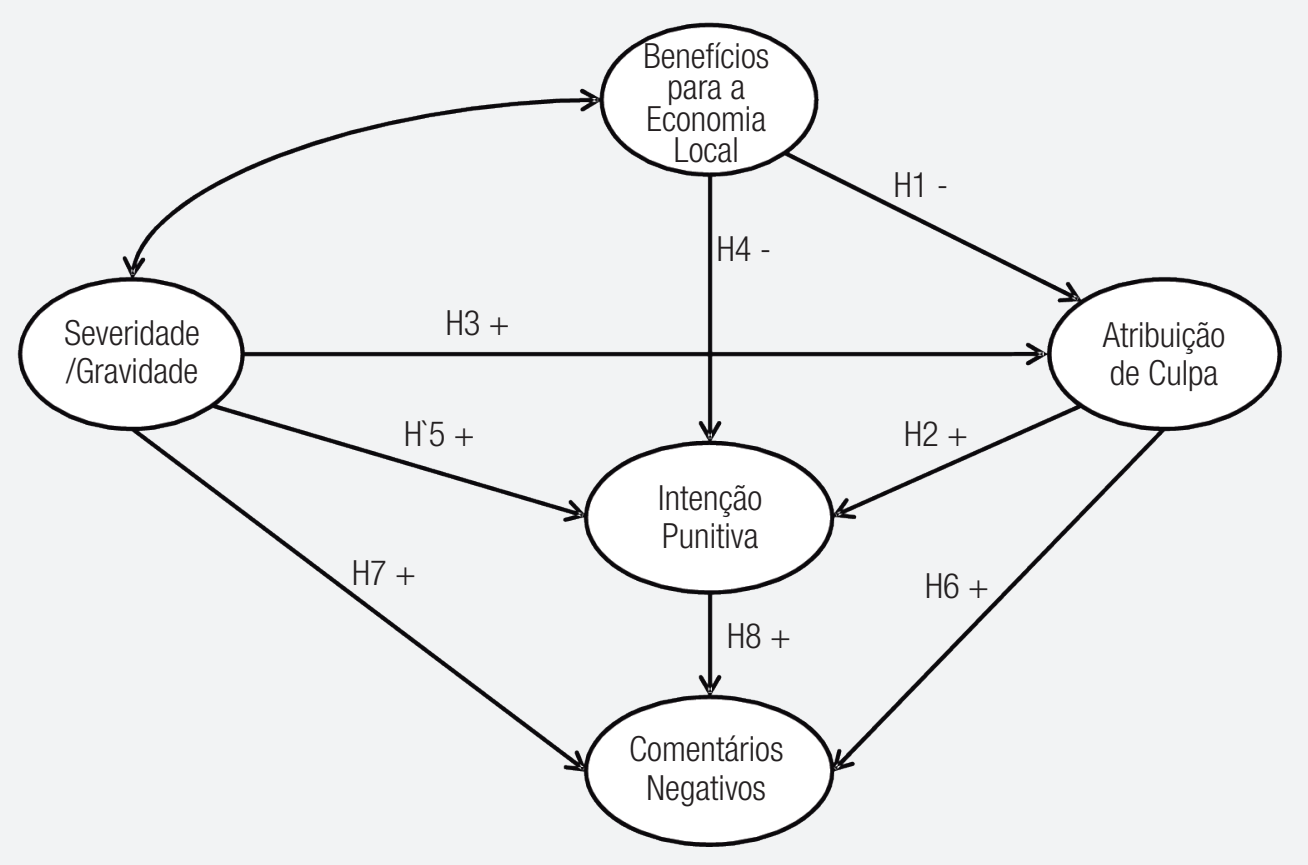

Fonte: Elaborada pelos autores.

\section{MÉTODO}

Os municípios incluídos na pesquisa foram: Anchieta-ES, Colatina-ES, Guarapari-ES, Linhares-ES e Mariana-MG. O motivo da escolha desses municípios se deu pela forma como cada um foi afetado. As cidades de Anchieta-ES e Guarapari-ES não sofreram impacto direto do desastre, dada sua localização mais ao sul do Estado do Espírito Santo, mas sofreram economicamente em função da Samarco Mineração S.A. ter suspendido suas atividades. Já as cidades de Colatina-ES e Linhares-ES, como estão ao norte do Espírito Santo e devido ao fato do Rio Doce as percorrer, sofreram o impacto ambiental do desastre, mas não eram beneficiadas economicamente pela mineradora. Já a cidade de Mariana-MG, onde se originou a tragédia, sofreu tanto ambiental quanto economicamente em função da paralisação da empresa (Augusto, 2016; Avila, 2015; Folha de S. Paulo, 2015; Gonçalves, Fusco, \& Vespa, 2016; Vignolli, 2016). Em complemento, a Tabela 1 apresenta detalhes do tipo de benefício e o efeito ambiental. 


\section{TABELA 1}

\section{CARACTERIZAÇÃO DAS CIDADES EM RELAÇÃO AO DESASTRE AMBIENTAL E À SAMARCO MINERAÇÃO S.A.}

\begin{tabular}{|c|c|c|c|c|c|c|}
\hline Municípios & $\begin{array}{c}\text { Tipo de benefícios } \\
\text { econômicos gerados } \\
\text { pela Samarco Mineração } \\
\text { S.A. }\end{array}$ & $\begin{array}{c}\text { Tipo de efeito } \\
\text { com o desastre } \\
\text { ambiental }\end{array}$ & Populaçãoa & $\begin{array}{l}\mathrm{PIBb}(\mathrm{RS} \\
\text { mil) }\end{array}$ & $\begin{array}{c}\text { PIB per } \\
\text { capitab (R\$) }\end{array}$ & $\begin{array}{c}\text { Principais atividades } \\
\text { econômicas }\end{array}$ \\
\hline Anchieta-ES & Alto & Efeito indireto & 28.546 & 2.758 .914 & $99.873,80$ & $\begin{array}{c}\text { Indústria (Mineração) } \\
\text { Agricultura familiar } \\
\text { Pecuária } \\
\text { Pesca }\end{array}$ \\
\hline Colatina-ES & Nenhum & Efeito direto & 124.525 & 3.194 .789 & $26.048,87$ & $\begin{array}{c}\text { Confecção } \\
\text { Agricultura } \\
\text { Granito }\end{array}$ \\
\hline Guarapari-ES & Médio & Efeito indireto & 123.166 & 2.041 .157 & $17.037,76$ & $\begin{array}{c}\text { Comércio, serviços e } \\
\text { turismo }\end{array}$ \\
\hline Linhares-ES & Nenhum & Efeito direto & 169.048 & 5.239 .007 & $32.011,14$ & $\begin{array}{c}\text { Indústria (moveleira), } \\
\text { Petróleo e gás, } \\
\text { Agricultura e } \\
\text { pecuária }\end{array}$ \\
\hline Mariana-MG & Alto & Efeito direto & 59.857 & 3.099 .191 & $52.705,53$ & $\begin{array}{l}\text { Mineração, turismo } \\
\text { cultural e comércio }\end{array}$ \\
\hline
\end{tabular}

Nota: a: Estimada para 2017; b: referente ao ano de 2015.

Fonte: Elaborada pelos autores.

Mesmo essas cidades tendo sofrido diferentes impactos, é preciso registrar que elas não foram as únicas afetadas, já que o desastre causou danos a todas as cidades de Minas Gerais e do Espírito Santo localizadas às margens do Rio Gualaxo do Norte, do Rio do Carmo e do Rio Doce. Estima-se que 40 municípios foram afetados (Instituto Brasileiro do Meio Ambiente e dos Recursos Naturais Renováveis, 2015). A Figura 2 demonstra a extensão do evento.

Para a aplicação da pesquisa, o estudo adotou um instrumento com escalas psicométricas que tornaram possível a mensuração de julgamentos e sentimentos que certos indivíduos apresentavam em relação a um ato de IrSE. Tais escalas foram previamente adotadas por Antonetti e Maklan (2016a, 2016b) e Grégoire, Laufer, e Tripp (2010). Como essas escalas foram originalmente desenvolvidas em inglês, elas passaram por um processo de tradução e adaptação transcultural que procurou seguir os padrões nacionais (Giusti \& Befi-Lopes, 2008) e internacionais (Sperber, 2004). O instrumento utilizado foi composto por 15 itens formulados como questões, sendo eles distribuídos em 4 escalas: "atribuição de culpa", "severidade/gravidade", "intenção punitiva" e "comentários negativos". 


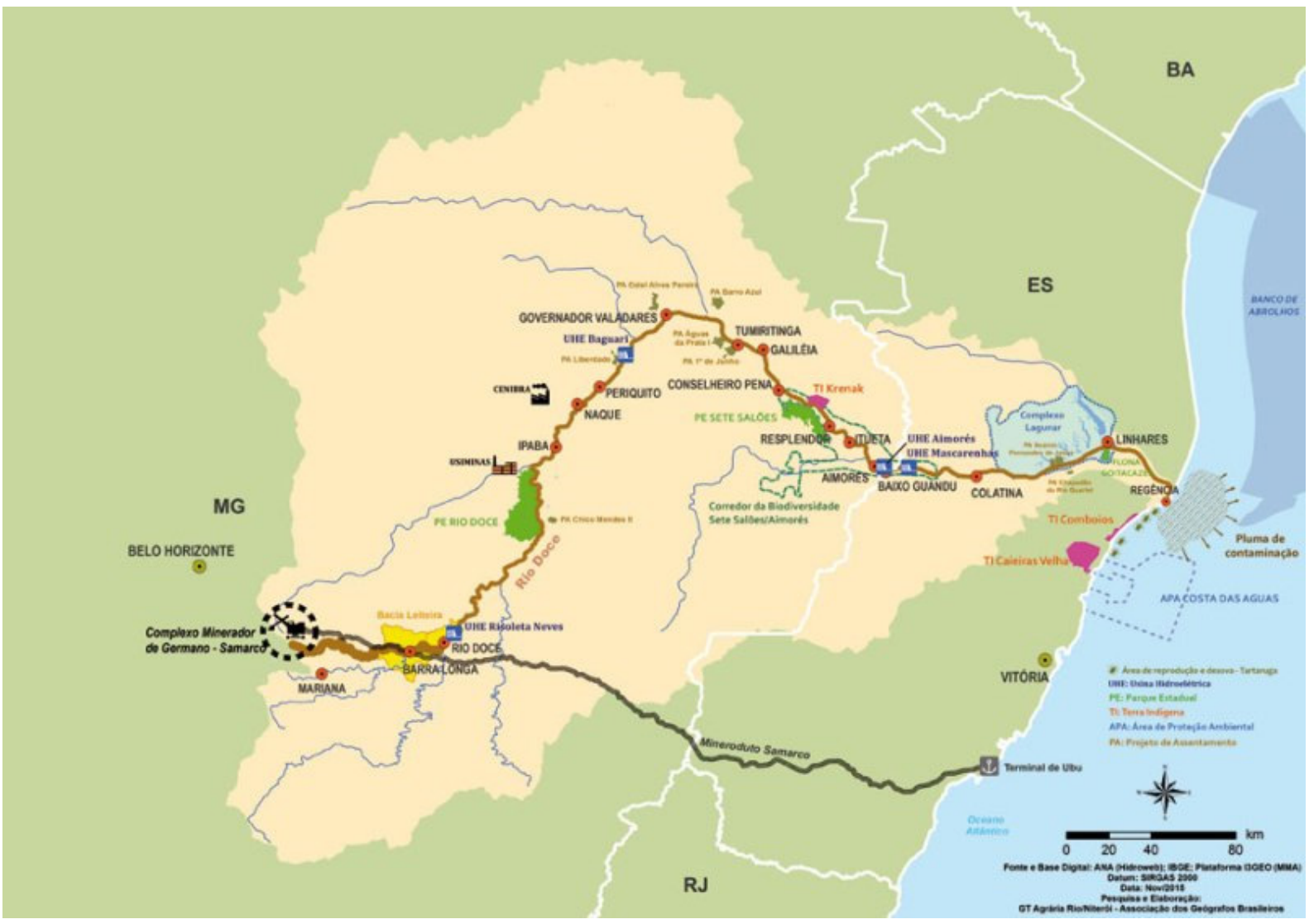

Fonte: Fundação Oswaldo Cruz (2016).

A validação psicométrica desse instrumento foi realizada em uma amostra composta por 249 estudantes universitários de uma faculdade em Guarapari-ES. A escolha dessa amostra foi baseada na conveniência, pelo critério de facilidade de acesso. A fatorabilidade dos dados foi atestada pelo indicador Kaiser-Meyer-Olkin Measure of Sampling Adequacy $(\mathrm{KMO}=0,91)$ e pelo Teste de Esfericidade de Bartlett (qui-quadrado $=3.979,14 ; p<0,001$ ) (Hair, Black, Babin, Anderson, \& Tatham, 2009). O alfa de Cronbach ( $\alpha$ ) ficou acima de $0,7 \mathrm{em}$ todas as escalas e as cargas fatoriais atenderam ao mínimo geralmente aceito, de 0,32 (Tabachnick \& Fidell, 2013).

Em complemento, buscou-se uma escala que mensurasse os "benefícios para a economia local" proporcionados pela Samarco Mineração S.A., sendo encontrada no trabalho de Lima (2012) e apresentando em sua composição 7 itens. Como essa escala foi desenvolvida em português, não foi necessário o processo de tradução.

A técnica estatística escolhida para testar o modelo foi a modelagem de equações estruturais, que permite analisar "uma série de relações de dependência simultaneamente. Ela é particularmente útil quando uma variável dependente se torna independente em relações subsequentes de dependência" (Hair et al., 2009, p. 539). Para a testagem das hipóteses foi utilizado o modelo proposto em cada uma das cinco cidades, sendo coletado um total de 1.616 questionários. A coleta de dados se concentrou em prefeituras, faculdades, institutos tecnológicos e escolas técnicas, além de solicitações de indicações de 
e-mail realizadas nos questionários e buscas em redes sociais. A Tabela 2 apresenta um detalhamento da amostra pesquisada.

\section{TABELA 2 QUANTIDADE DE INDIVÍDUOS POR CIDADE}

\begin{tabular}{lccccc} 
Cidades & $\begin{array}{c}\text { Quantidade total } \\
\text { de indivíduos } \\
\text { pesquisados }\end{array}$ & $\begin{array}{c}\text { Quantidade de indivíduos } \\
\text { excluídos da amostra em } \\
\text { função de dados incompletos }\end{array}$ & $\begin{array}{c}\text { Quantidade final } \\
\text { de indivíduos } \\
\text { pesquisados }\end{array}$ & $\begin{array}{c}\text { Quantidade de } \\
\text { homens }\end{array}$ & $\begin{array}{c}\text { Quantidade de } \\
\text { mulheres }\end{array}$ \\
\hline Anchieta-ES & 296 & 4 & 292 & 145 & 147 \\
Colatina-ES & 343 & 6 & 337 & 139 & 198 \\
Guarapari-ES & 284 & 3 & 281 & 132 & 149 \\
Linhares-ES & 389 & 13 & 376 & 127 & 249 \\
Mariana-MG & 335 & 5 & 330 & 154 & 175 \\
Total & 1.647 & 31 & 1.616 & $697^{\star}$ & $918^{*}$
\end{tabular}

* Entre os indivíduos participantes da pesquisa, um deles alegou que não se classificava nem como homem nem como mulher, por isso, não foi incluído na quantidade de homens e mulheres listada.

Fonte: Elaborada pelos autores.

Na modelagem por equações estruturais foi utilizado o método de estimação Weighted Least Squares Robust (WLSMV), em função da natureza ordinal dos itens. Após a especificação e estimação do modelo, seu ajuste foi avaliado por meio dos seguintes indicadores: qui-quadrado, Tucker-Lewis Index (TLI), Comparative Fit Index (CFI) e Root Mean Square Error of Approximation (RMSEA). O qui-quadrado $\left(\chi^{2}\right)$, como critério, não deveria ser significativo e, caso fosse, adotamos o parâmetro da razão do qui-quadrado pelos graus de liberdade inferior a 5,0. O TLI e o CFI deveriam apresentar valores idealmente superiores a 0,95 , sendo aceitáveis valores acima de 0,90 para indicar a adequação do modelo aos dados. Por fim, considerou-se o ponto de corte inferior a 0,06 do índice de parcimônia do modelo - RMSEA (Hu \& Bentler, 1999).

\section{RESULTADOS}

A análise exploratória dos dados identificou poucos dados omissos (missing values), de caráter aleatório, que foram substituídos pela média e em alguns casos, sendo esta arredondada para as categorias imediatamente posteriores ou anteriores, evitando a criação de novas categorias. A análise descritiva apontou escores que se aproximaram de uma curva normal.

Após as análises exploratórias foram testados os modelos de estrutura latente para cada uma das cidades pesquisadas. Assim, uma análise inicial da modelagem de equações estruturais evidenciou que o modelo não teve um bom ajuste em nenhuma das cidades. Buscou-se, então, verificar as alterações sugeridas pelos Modification Indices (MI), sendo constatadas oito correlações entre erros de itens das mesmas escalas. Ao realizar a análise de cada uma das modificações sugeridas, identificou-se que a correlação dos erros estava vinculada à semelhança no conteúdo de alguns itens. Desse modo, após 
os ajustes sugeridos pelo MI, o modelo passou a apresentar os índices de ajuste aceitáveis, conforme a Tabela 3. Considerando essa nova apresentação de acordo com o modelo ajustado, evidenciam-se na Tabela 4 os resultados da modelagem de equações estruturais.

\section{TABELA 3 ÍNDICES DO MODELO AJUSTADO ÀS CORRELAÇÕES DOS ERROS}

\begin{tabular}{|c|c|c|c|c|c|c|c|c|}
\hline Cidades & $\begin{array}{l}\text { Número de } \\
\text { observações } \\
\text { (N) }\end{array}$ & $\chi^{2}(172)$ & $\chi^{2} / \mathrm{df}$ & RMSEA & $\begin{array}{c}90 \% \mathrm{Cl} \text {, } \\
\text { limite } \\
\text { inferior }\end{array}$ & $\begin{array}{l}\text { Limite } \\
\text { superior }\end{array}$ & CFI & TLI \\
\hline Anchieta-ES & 292 & $362,779^{* * *}$ & 2,109 & 0,062 & 0,053 & 0,070 & 0,950 & 0,938 \\
\hline Guarapari-ES & 281 & $340,952^{\star \star \star}$ & 1,982 & 0,059 & 0,050 & 0,068 & 0,950 & 0,939 \\
\hline Mariana-MG & 330 & $325,015^{\star \star \star}$ & 1,890 & 0,052 & 0,043 & 0,061 & 0,969 & 0,963 \\
\hline Colatina-ES & 337 & $349,371^{\star \star *}$ & 2,031 & 0,055 & 0,047 & 0,064 & 0,951 & 0,940 \\
\hline Linhares-ES & 376 & $415,371^{\star \star *}$ & 2,415 & 0,061 & 0,054 & 0,069 & 0,937 & 0,923 \\
\hline
\end{tabular}

Nota: ${ }^{* *} p<0,001 ; \chi^{2}=$ qui-quadrado; $\mathrm{df}=$ graus de liberdade; RMSEA $=$ Root Mean Square Error of Approximation; $\mathrm{CFI}=$ Comparative Fit Index; TLI $=$ Tucker-Lewis Index.

Fonte: Elaborada pelos autores.

TABELA 4 RESULTADO DA MODELAGEM DE EQUAÇÕES ESTRUTURAIS PARA 0 MODELO AJUSTADO

\begin{tabular}{|c|c|c|c|c|c|}
\hline Construtos (coeficientes padronizados) & Anchieta & Guarapari & Mariana & Colatina & Linhares \\
\hline \multicolumn{6}{|l|}{ Modelo estrutural } \\
\hline Atrib. Culpa - > Intenção Punitiva (H2+) & $-0,352$ & $-0,236$ & 0,245 & 0,247 & 0,228 \\
\hline Benef. Economia Local -> Int. Punitiva (H4-) & $-0,424^{\star \star \star}$ & $-0,252^{\star \star \star}$ & $-0,294^{\star \star \star}$ & $-0,048$ & $-0,025$ \\
\hline Severidade/Gravidade -> Int. Punitiva (H5+) & 0,691 & 0,633 & 0,252 & 0,242 & 0,3127 \\
\hline Benef. Economia Local -> Atrib. Culpa (H1-) & $-0,037$ & 0,030 & $-0,034$ & $-0,032$ & $-0,062$ \\
\hline Severidade/Gravidade -> Atrib. Culpa (H3+) & $0,951^{\star \star \star}$ & $0,949^{\star \star \star}$ & $0,945^{\star \star \star}$ & $0,910^{\star \star \star}$ & $0,902^{\star \star *}$ \\
\hline Int. Punitiva - > Comentários Negativos (H8+) & $0,786^{\star \star \star}$ & $0,923^{\star \star \star}$ & $0,972^{\star \star \star}$ & $0,819^{\star \star \star}$ & $0,829^{\star * *}$ \\
\hline Atrib.Culpa - > Comentários Negativos (H6+) & $-0,028$ & $-0,045$ & $-0,249$ & $-0,357$ & $-0,300$ \\
\hline Severidade/Gravidade - > C. Negativos $(\mathrm{H} 7+)$ & 0,046 & 0,075 & 0,246 & 0,378 & 0,347 \\
\hline Número de observações & 292 & 281 & 330 & 337 & 376 \\
\hline
\end{tabular}

Nota: ${ }^{* * *} p<0,001$.

Fonte: Elaborada pelos autores.

A análise mostra que nem todas as hipóteses de pesquisa foram confirmadas. Duas hipóteses (H3 e H8) foram suportadas nas cinco cidades pesquisadas, enquanto outra (H4) foi suportada somente em 
Anchieta-ES, Guarapari-ES e Mariana-MG. As demais hipóteses de pesquisa não foram suportadas. Esses resultados serão discutidos na próxima seção, à luz da literatura.

\section{DISCUSSÃO}

Este estudo propõe reflexões sobre como a identificação social advinda de benefícios econômicos pode atenuar certos sentimentos e intenções para com a empresa, de forma a não a responsabilizar e não intencionar puni-la pelos danos causados. O trabalho explorou a IrSE, abordando o desastre causado pela Samarco Mineração S.A. ao município de Mariana-MG, com o objetivo de investigar se a identificação social com a empresa, que surge em função dos benefícios econômicos, reduz a intenção punitiva e a atribuição de culpa. De maneira sintética, pode-se dizer que, para as cidades pesquisadas, a severidade aumentou a atribuição de culpa e a intenção punitiva aumentou a intenção de fazer comentários negativos. Além disso, os benefícios para a economia local reduziram a intenção punitiva dos moradores de Anchieta-ES, Guarapari-ES e Mariana-MG. Portanto, nas cidades onde a empresa gerou benefícios, as pessoas tinham menor tendência a puni-la. Tal realidade é instigante, pois não incentiva a empresa causadora do dano, bem como suas controladoras, a tomar medidas que possam evitar eventuais desastres futuros, como o ocorrido em 25 de janeiro de 2019 na cidade de Brumadinho-MG, cuja empresa envolvida foi a Vale S.A.(O Portal de Notícias da Globo, 2019b; Schreiber, 2019).

Nesse contexto, merece menção o fato da hipótese $\mathrm{H} 3$ ter sido comprovada, demonstrando que a severidade/gravidade do evento aumentou a atribuição de culpa nas cidades pesquisadas. Isso evidenciou que, mesmo em municípios onde a empresa gerava benefícios, essa relação positiva não deixou de existir.

Ademais, a atribuição de culpa está relacionada à percepção de severidade/gravidade de determinado evento (Kelley \& Michela, 1980), de tal sorte que os danos causados pelas empresas que agirem com IrSE podem gerar emoções negativas nas pessoas. Tais emoções são, portanto, influenciadas pela severidade atribuída ao evento (Antonetti \& Maklan, 2016a; Kelley \& Michela, 1980).

Outra hipótese que foi suportada foi a H4, cujo suporte foi obtido em Anchieta-ES, Guarapari-ES e Mariana-MG, apontando que, quando a empresa gera benefícios, os indivíduos tendem a uma menor intenção de puni-la. Inclusive, essa relação demonstrou ser mais forte em Anchieta-ES, tendo em vista que a prefeitura da cidade mostrou que sua maior fonte de arrecadação tributária vinha da mineradora (Prefeitura Municipal de Anchieta, 2017).

Já em relação à cidade de Guarapari-ES, esta apresentou o menor coeficiente. Tal resultado levou à inferência de que a relação negativa dos benefícios à economia local com a intenção punitiva tem menor efeito nessa cidade. Por outro lado, chamou atenção o fato dessa hipótese obter suporte na cidade de Mariana-MG, tendo em vista que foi afetada diretamente pelo desastre ambiental, causando destruição e mortes. Essa evidência apontou que, mesmo diante de um evento dessas proporções, os benefícios que a Samarco Mineração S.A. gerava reduziram a intenção de puni-la.

Esses resultados estão em linha com os apresentados por Lange e Washburn (2012), ao argumentar que, quando existe identificação social de indivíduos com uma firma, tal identificação pode influenciar minimizando reações contrárias a IrSE. Portanto, como se esperava previamente, os moradores de Anchieta-ES, Guarapari-ES e Mariana-MG tenderam a uma menor intenção de punir a empresa em função de apresentarem uma identificação social com a mineradora, dados os benefícios econômicos. 
Já para Colatina-ES e Linhares-ES, os resultados também foram congruentes com as expectativas, tendo em vista que não se esperava essa identidade social com a mineradora, já que tais cidades não se beneficiavam diretamente com a empresa, percebendo apenas os impactos do desastre.

A terceira hipótese suportada foi a $\mathrm{H} 8$, sendo tal relação positiva evidenciada nas cinco localidades, confirmando que a intenção de punir aumentou a intenção de fazer comentários negativos. No que se refere às hipóteses H1, H2, H5, H6 e H7, elas não foram suportadas. Essa constatação demonstra que, mesmo a literatura prevendo a relação entre as variáveis estudadas, tais apontamentos não encontraram suporte estatístico para o caso estudado. Essas evidências estatísticas demonstram que, apesar dos benefícios para a economia local influenciarem negativamente a intenção punitiva nas cidades de Anchieta-ES, Guarapari-ES e Mariana-MG, não foram encontradas evidências de que esses benefícios influenciaram negativamente a atribuição de culpa (H1). Esse achado apontou que, mesmo em cidades beneficiadas pela empresa, os indivíduos não reduziram a atribuição de culpa. Ademais, a própria empresa não se isentou da responsabilidade diante do ocorrido.

A respeito de tais resultados, é preciso retomar ao processo de atribuição de culpa, dado que, para apontar um culpado, há necessidade de uma atribuição por parte dos grupos sociais (Lange \& Washburn, 2012). Diante disso, a atribuição de culpa se torna negativamente menor quando o observador tem identificação social com a firma causadora do dano. Mas não foi isso que ocorreu no caso da Samarco Mineração S.A., pois os moradores atribuíram culpa à empresa.

Todavia, chamou atenção o fato de que em nenhuma das cidades a atribuição de culpa afetou positivamente a intenção punitiva $(\mathrm{H} 2)$. Apesar do referencial teórico apontar indícios dessa relação, ela não foi suportada na pesquisa empírica. Entretanto, alguns autores apontam que, em vários países em desenvolvimento, as pessoas se mostram menos propensas a penalizar as empresas, tendo em vista os benefícios que elas proporcionaram (Bazerman \& Tenbrunsel, 2011; Hilson, 2012). Aliás, também se constatou que a avaliação da severidade/gravidade do desastre não influenciou positivamente a intenção punitiva (H5). Esse achado se associa com a condição social e econômica do Brasil, dado que países nessa condição foram menos propensos a punir a empresa.

Em meio a essa realidade, inferiu-se que os diferentes grupos sociais podem perceber de forma diferente um ato de IrSE. Considerando esses grupos como os moradores das cidades investigadas, observa-se que eles foram impactados de forma diferenciada. Outrossim, esses moradores percebem a empresa causadora do evento de forma diferente, tendo em vista que para alguns a empresa gerava benefícios e para outros, não. Sendo assim, as visões de grupos da sociedade em relação a uma corporação podem estar desalinhadas, sobretudo quando a empresa traz certos benefícios (Hogg et al., 1995; Scherer \& Palazzo, 2007).

Assim, considerando que as percepções de injustiça ou iniquidade são afetadas em função da identidade social que surge dos benefícios gerados, a intenção punitiva também poderia ser afetada ao considerar que ela só ocorreria se a sociedade perceber que a empresa cometeu alguma injustiça. Cabe ressaltar o fato da dimensão atribuição de culpa não influenciar positivamente a dimensão comentários negativos (H6). Essa característica não está alinhada com os achados teóricos e demonstrou um comportamento passivo da sociedade brasileira perante a influência econômica da empresa. Por conseguinte, a dimensão severidade/gravidade (H7) não influenciou positivamente a dimensão comentários negativos. Essa observação demonstra que a amostra apresentou um comportamento diferente daquele que foi constatado por Antonetti e Maklan (2016a), tendo em vista que essa hipótese foi por eles suportada. Esse resultado possibilita inferir que esse seja um comportamento que pode repetir-se em outros locais do Brasil, o que caracterizaria uma conduta diferente de outros países. 
Diante do exposto, é possível inferir que o benefício econômico da empresa impactou pouco a atribuição de culpa, mas trouxe efeito na intenção punitiva. Esse achado complementa os resultados apontados por Antonetti e Maklan (2016a, 2016b), tendo em vista que não exploraram os efeitos dos benefícios econômicos sobre a atribuição de culpa e a intenção punitiva.

Assim como no caso da Shell, que derramou petróleo no Delta da Nigéria durante décadas, mas não teve suas atividades suspensas em função do impacto no PIB nigeriano (George et al., 2012), tal situação se repete em relação ao caso do desastre ambiental de Mariana-MG, dado que, logo após o evento, a Samarco Mineração S.A. deixou clara sua contribuição financeira para os estados de Minas Gerais e Espírito Santo (Samarco Mineração S.A., 2016). Inclusive, essa informação se confirmou ao constatar a queda de 3,9\% do PIB do Espírito Santo, além da redução do PIB mineiro, sendo esses fatos atribuídos à paralisação da Samarco Mineração S.A. (Fundação João Pinheiro, 2016; O Portal de Notícias da Globo, 2016). Sendo assim, os estados afetados não sofreram somente com a lama que foi derramada, mas também com o impacto econômico que a paralisação da companhia trouxe.

Nesse sentido, é compreensível um resultado que aponta a atribuição de culpa à Samarco Mineração S.A., mas tal atribuição não influencia os comentários negativos e a intenção punitiva. Desse modo, se por um lado há o reconhecimento dos moradores das cinco cidades de que a empresa foi culpada pelos danos, por outro não há intenção de puni-la, possivelmente devido ao receio de que isso venha a afetar a economia, como já aconteceu em outros acidentes ambientais pelo mundo (George et al., 2012; Hilson, 2012). Essa realidade pode ter influenciado tanto a empresa quanto suas controladoras, Vale S.A. e BHP Billiton, a não tomarem medidas visando a evitar futuros desastres como o ocorrido em janeiro de 2019 em Brumadinho-MG.

\section{CONCLUSÃO}

Apesar da temática IrSE apresentar discussões há anos, esse assunto costuma não ser discutido de forma explícita na literatura sobre RSE (Lange \& Washburn, 2012). Inclusive, a literatura nacional demonstrou que pouco se tem dedicado ao conteúdo, fato este que instigou a realização de uma pesquisa. Diante disso, o trabalho teve por objetivo constatar se a identificação social com a Samarco Mineração S.A., que surge em função dos benefícios econômicos, reduz a intenção punitiva de um evento e a atribuição de culpa dessa empresa.

Diante dos resultados, conclui-se que nas cidades onde a mineradora gerava empregos e beneficiava a economia, isto é, Anchieta-ES, Guarapari-ES e Mariana-MG, a população teve uma percepção maior de benefícios, afetando negativamente a intenção punitiva em relação à empresa. Por outro lado, a percepção de benefícios para a economia nessas três cidades não afetou negativamente a atribuição de culpa. Uma das explicações dessa constatação se deve ao fato da empresa, ao fazer uso de estratégias de gerenciamento de impressão, não procurou se eximir da responsabilidade, todavia, isso deixou claro o quanto contribuía para a economia do Espírito Santo e de Minas Gerais, além das ações que seriam tomadas (Samarco Mineração S.A., 2016, 2018).

Ademais, a atribuição de culpa por parte dos moradores das cinco cidades pesquisadas não afetou positivamente a intenção de punir a empresa. Destaca-se que a percepção brasileira quanto à punição é um pouco prejudicada em função de uma descrença no sistema judiciário nacional (Reichenheim et al., 2011). E os países em desenvolvimento procuram dar pouca atenção a problemas ligados à poluição (Surroca, Tribo, \& Zahra, 2013). 
Quanto à avaliação de severidade/gravidade do evento, essa percepção afetou positivamente a atribuição de culpa da empresa, mesmo confrontando os resultados com localidades onde a Samarco Mineração S.A. era economicamente importante. Por sua vez, não foram encontradas evidências de que a avaliação de severidade do evento afetasse positivamente a intenção de punir a empresa. Apesar de existirem evidências teóricas que relataram a existência dessa relação, o mesmo não foi confirmado pela pesquisa.

Por conseguinte, não foram encontradas evidências de que a atribuição de culpa da empresa por parte dos moradores das cidades pesquisadas afetava positivamente a intenção de fazer comentários negativos à mineradora. Cabe destacar que, se a atribuição de culpa não afetou positivamente a intenção punitiva, era de se esperar que a atribuição de culpa não provocasse efeitos sobre a intenção de fazer comentários negativos, isso considerando que o propósito de fazer comentários negativos também pode ser encarado como uma punição (Buttle, 1998). Ademais, a avaliação de severidade/ gravidade também não afetou positivamente a intenção de fazer comentários negativos à empresa.

Na sequência, constatou-se que a intenção punitiva por parte dos moradores das cidades afetou positivamente a intenção de fazer comentários negativos à empresa. Ao apurar os resultados, verificouse que eles são compatíveis com outras evidências teóricas (Buttle, 1998). Todos esses achados abriram espaço para a realização de outras pesquisas que busquem avaliar sentimentos, julgamentos e atribuições que possam ser oriundos de desastres ocasionados por empresas.

Todavia, cabe destacar algumas limitações da pesquisa. Considerando que a investigação foi realizada em um único momento, esse fato pode ter aumentado a possibilidade de viés nos resultados. Outro ponto é que as avaliações estão restritas a um único evento, não possibilitando generalizações. Também se identificou que a amostra representa a opinião de cinco cidades, não sendo possível inferir esses resultados para outras localidades.

Tomando como base o apresentado e considerando que a temática da IrSE ainda é recente no Brasil, sugere-se uma agenda mais ampla para futuras pesquisas. A princípio, recomenda-se uma investigação qualitativa, fazendo uso de entrevistas ou grupos de discussão focal, buscando confrontar os resultados destacados, o que proporcionará melhor entendimento sobre o assunto. Além disso, considerando os resultados obtidos, a futura pesquisa qualitativa poderia discutir a dificuldade de entendimento de culpa e punição, tendo em vista que nas cidades de Anchieta-ES, Guarapari-ES e Mariana-MG os benefícios proporcionados pela Samarco Mineração influenciavam negativamente a intenção de punir a empresa. Para além desse ponto, essa pesquisa qualitativa também poderia ter como foco o debate sobre se a empresa teve ou não culpa e se deve ser punida ou não. Também se poderia abordar qual tipo de punição deve ser adotada.

Estudos quantitativos com outros eventos e empresas acerca da IrSE também seriam úteis para testar a estrutura de configuração das medidas aqui utilizadas. Além disso, outros preditores do julgamento da população acerca dos atos de IrSE também poderiam ser testados, tais como grau de escolaridade, nível de dano oriundo do evento, idade e renda familiar. Sugerem-se, ainda, estudos que continuem investigando atos de IrSE por outras empresas ou em outras localidades, sendo possível apontar o ocorrido em 2019 em Brumadinho-MG, possibilitando comparar os novos resultados com aqueles aqui evidenciados. Recomenda-se, por fim, a realização de estudos longitudinais que permitam acompanhar como o julgamento da população acerca dos atos de IrSE evolui à medida que o evento-chave se distancia. 


\section{REFERÊNCIAS}

Alexander, P. B. (2015). Corporate social irresponsibility. New York, NY: Routledge.

Antonetti, P., \& Maklan, S. (2016a). An Extended Model of Moral Outrage at Corporate Social Irresponsibility. Journal of Business Ethics, 135(3), 429-444.

Antonetti, P., \& Maklan, S. (2016b). Social identification and corporate irresponsibility: a model of stakeholder punitive intentions. British Journal of Management, 27(3), 583-605.

Armstrong, J. S. (1977). Social irresponsibility in management. Journal of Business Research, 5(3), $185-213$

Augusto, L. (2016). Prefeito de Mariana se reúne com Temer para pedir volta da Samarco. Recuperado de http://brasil.estadao.com.br/noticias/geral,prefeitode-mariana-se-reune-com-temer-para-pedirretorno-da-samarco-a-cidade, 10000054576

Avila, G. (2015). Guarapari pode sofrer impacto de $R \$$ 12 milhões com paralisação da Samarco. Recuperado de https://www.portal27.com.br/guarapari-podesofrer-impacto-de-r-12-milhoes-com-paralisacaoda-samarco/

Bazerman, M. H., \& Tenbrunsel, A. E. (2011). Blind spots: why we fail to do what's right and what to do about it. Princeton, NJ: Princeton University Press.

Berger, P. L., \& Luckmann, T. (1985). Construção social da realidade: tratado de sociologia do conhecimento. Petrópolis, RJ: Vozes.

Buttle, F. A. (1998). Word of mouth: understanding and managing referral marketing. Journal of Strategic Marketing, 6(3), 241-254.

Campbell, M. C. (1995). When attention-getting advertising tactics elicit consumer inferences of manipulative intent: the importance of balancing benefits and investments. Journal of Consumer Psychology, 4(3), 225-254.

Carlsmith, K. M., Darley, J. M., \& Robinson, P. H. (2002). Why do we punish? Deterrence and just deserts as motives for punishment. Journal of Personality and Social Psychology, 83(2), 284-299.

Chang, H. H., Tsai, Y.-C., Wong, K. H., Wang, J. W., \& Cho, F. J. (2015). The effects of response strategies and severity of failure on consumer attribution with regard to negative word-of-mouth. Decision Support Systems, 71, 48-61.

Chiu, S.-C., \& Sharfman, M. (2018). Corporate social irresponsibility and executive succession: an empirical examination. Journal of Business Ethics, 149(3), 707-723.

Dunford, L., \& Ridley, A. (1996). No soul to be damned, no body to be kicked": responsibility, blame and corporate punishment. International Journal of the Sociology of Law, 24, 1-19. Recuperado de http://www.sciencedirect.com/science/article/pii/ S0194659596900015

Folha de S. Paulo. (2015). O caminho da lama. Recuperado de http://temas.folha.uol.com.br/ocaminho-da-lama/

Fundação João Pinheiro. (2016). PIB de Minas Gerais expande 0,1\% no segundo trimestre de 2016. Recuperado de http://www.fjp.mg.gov.br/index.php/ noticias-em-destaque/3644-pib-de-minas-geraisexpande-0-1-no-segundo-trimestre-de-2016

Fundação Osvaldo Cruz. (2016). Cenário de fim do mundo no rastro da lama. Recuperado de http:// www.epsjv.fiocruz.br/noticias/reportagem/cenariode-fim-do-mundo-no-rastro-da-lama

George, O. J., Kuye, O. L., \& Onokala, U. C. (2012). Corporate social irresponsibility (CSI) a catalyst to the Niger Delta crisis: the case of Nigerian oil multinational companies versus the militants of Niger Delta Region of Nigeria. Journal of Management Research, 4(2), 1-11.

Giusti, E., \& Befi-Lopes, D. M. (2008). Tradução e adaptação transcultural de instrumentos estrangeiros para o português brasileiro (PB). Pró-Fono Revista de Atualização Científica, 20(3), 207-210.

Gonçalves, E., Fusco, N., \& Vespa, T. (2016). Tragédia em Mariana: para que não se repita. Recuperado de http://veja.abril.com.br/complemento/brasil/paraque-nao-se-repita/

Grégoire, Y., Laufer, D., \& Tripp, T. M. (2010). A comprehensive model of customer direct and indirect revenge: understanding the effects of perceived greed and customer power. Journal of the Academy of Marketing Science, 38(6), 738-758. 
Hair, J. F., Jr., Black, W. C., Babin, B. J., Anderson, R. E., \& Tatham, R. L. (2009). Análise multivariada de dados (6th ed.). Porto Alegre, RS: Bookman.

Hamilton, V. L. (1980). Intuitive psychologist or intuitive lawyer? Alternative models of the attribution process. Journal of Personality and Social Psychology, 39(5), 767-772.

Hilson, G. (2012). Corporate social responsibility in the extractive industries: experiences from developing countries. Resources Policy, 37(2), 131137.

Hogg, M. A., Terry, D. J., \& White, K. M. (1995). A tale of two theories: a critical comparison of identity theory with social identity theory. Social Psychology Quarterly, 58(4), 255-269.

Hu, L., \& Bentler, P. M. (1999). Cutoff criteria for fit indexes in covariance structure analysis: conventional criteria versus new alternatives. Structural Equation Modeling: A Multidisciplinary Journal, 6(1), 1-55.

Instituto Brasileiro do Meio Ambiente e dos Recursos Naturais Renováveis. (2015). Laudo técnico preliminar: impactos ambientais decorrentes do desastre envolvendo o rompimento da barragem de Fundão, em Mariana, Minas Gerais. Recuperado de http://www.ibama.gov.br/phocadownload/noticias_ ambientais/laudo_tecnico_preliminar_Ibama.pdf

Kang, C., Germann, F., \& Grewal, R. (2016). Washing away your sins? Corporate social responsibility, corporate social irresponsibility, and firm performance. Journal of Marketing, 80(2), 59-79.

Kelley, H. H., \& Michela, J. L. (1980). Attribution theory and research. Annual Review of Psychology, 31(1), 457-501.

Kotchen, M., \& Moon, J. J. (2012). Corporate social responsibility for irresponsibility. The B.E. Journal of Economic Analysis \& Policy, 12(1), 1-21.

Lange, D., \& Washburn, N. T. (2012). Understanding attributions of corporate social irresponsibility. Academy of Management Review, 37(2), 300-326.

Lee, Y.-C., Lu, Y.-C., \& Wang, Y.-C. (2019). Corporate social irresponsibility, CEO overconfidence, and stock price crash risk. Applied Economics Letters, 26(14), 1143-1147.

Lima, S. C. M. (2012). As perceções dos residentes do papel do turismo no desenvolvimento da Ilha da
Boavista. Recuperado de https://estudogeral.sib. uc.pt/bitstream/10316/19786/4/As perceções dos residentes do papel do turismo.pdf

Lin-Hi, N., \& Müller, K. (2013). The CSR bottom line: preventing corporate social irresponsibility. Journal of Business Research, 66(10), 1928-1936.

Martínez-Alier, J. (1995). The environment as a luxury good or "too poor to be green"? Ecological Economics, 13(1), 1-10.

McArthur, L. Z., \& Ginsberg, E. (1981). Causal attribution to salient stimuli: an investigation of visual fixation mediators. Personality and Social Psychology Bulletin, 7(4), 547-553.

Murphy, P. E., \& Schlegelmilch, B. B. (2013). Corporate social responsibility and corporate social irresponsibility: introduction to a special topic section. Journal of Business Research, 66(10), 1807-1813.

Nordgren, L. F., \& McDonnell, M.-H. M. (2010). The scope-severity paradox: why doing more harm is judged to be less harmful. Social Psychological and Personality Science, 2(1), 97-102.

O Portal de Notícias da Globo. (2016, 20 de junho). PIB cai 3,9\% no $2^{\circ}$ trimestre e ES liga resultado a Samarco e seca. Recuperado de http://g1.globo.com/ espirito-santo/noticia/2016/09/pib-cai-39-no-2trimestre-e-es-liga-resultado-samarco-e-seca.html

O Portal de Notícias da Globo. (2019a, 25 de janeiro). Rompimento de barragem de rejeitos de minério provoca desastre em Brumadinho (MG). Recuperado de https://g1.globo.com/ jornal-nacional/noticia/2019/01/25/rompimentode-barragem-de-rejeitos-de-minerio-provocadesastre-em-brumadinho-em-minas-gerais.ghtml

O Portal de Notícias da Globo. (2019b, 10 de abril). Sobe para 225 o número de mortos identificados no desastre da Vale em Brumadinho. Recuperado de https://g1.globo.com/mg/minas-gerais/ noticia/2019/04/10/sobe-para-225-o-numero-demortos-identificados-no-desastre-da-vale-embrumadinho.ghtml

Oh, W.-Y., Chang, Y. K., \& Jung, R. (2018). Experience-based human capital or fixed paradigm problem? CEO tenure, contextual influences, and corporate social (ir)responsibility. Journal of Business Research, 90, 325-333. 
Olofsson, J., \& Söderholm, L. (2014). The effect of corporations' irresponsible actions on young consumers' purchasing behavior in the FMCG apparel industry. Recuperado de http://www.diva-portal. org/smash/get/diva2:728818/FULLTEXT03.pdf

Ping, Q., Ishaq, M., \& Li, C. (2015). Product harm crisis, attribution of blame and decision making: an insight from the past. Journal of Applied Environmental and Biological Sciences, 5(5), 35-44.

Popa, M., \& Salanță, I. (2014). Corporate social responsibility versus corporate social irresponsibility. Management and Marketing. Challenges for the Knowledge Society, 9(2), 137-146. Recuperado de http://www.managementmarketing.ro/pdf/ articole/446.pdf

Prefeitura Municipal de Anchieta. (2017). História e perfil de Anchieta. Recuperado de http://www. anchieta.es.gov.br/materia_especifica/6495/historiae-perfil-de-anchieta

Reichenheim, M. E., Souza, E. R. de, Moraes, C. L., Jorge, M. H. P. de M., Silva, C. M. F. P. da, \& Minayo, M. C. de S. (2011). Violence and injuries in Brazil: the effect, progress made, and challenges ahead. The Lancet, 377(9781), 1962-1975.

Richins, M. L. (1983). Negative word-of-mouth by dissatisfied consumers: a pilot study. Journal of Marketing, 47(1), 68-78.

Richins, M. L. (1987). A multivariate analysis of responses to dissatisfaction. Journal of the Academy of Marketing Science, 15(3), 24-31.

Samarco Mineração S.A. (2016). Dossiê Samarco. Samarco Mineração S.A. Recuperado de http:// www.samarco.com/wp-content/uploads/2015/12/ Dossie_reduzido_19_05.pdf

Samarco Mineração S.A. (2018). Entenda o rompimento. Recuperado de http://www.samarco. com/rompimento-de-fundao/

Scherer, A. G., \& Palazzo, G. (2007). Toward a political conception of corporate responsibility: business and society seen from a Habermasian perspective. Academy of Management Review, 32(4), 1096-1120.

Schreiber, M. (2019). Tragédia em Brumadinho: quase três anos após desastre de Mariana, Vale ofereceu R \$ 30 mi em bônus recorde a seis diretores executivos. Recuperado de https://www.bbc.com/ portuguese/brasil-47095802

Schwartz, M. S., \& Carroll, A. B. (2003). Corporate social responsibility: a three-domain approach. Business Ethics Quarterly, 13(4), 503-530.

Schwarze, S. (2003). Corporate-state irresponsibility, critical publicity, and asbestos exposure in Libby, Montana. Management Communication Quarterly, 16(4), 625-632.

Sperber, A. D. (2004). Translation and validation of study instruments for cross-cultural research. Gastroenterology, 126(1), S124-S128.

Surroca, J., Tribo, J. A., \& Zahra, S. A. (2013). Stakeholder pressure on MNEs and the transfer of socially irresponsible practices to subsidiaries. Academy of Management Journal, 56(2), 549-572.

Sweetin, V. H., Knowles, L. L., Summey, J. H., \& McQueen, K. S. (2013). Willingness-to-punish the corporate brand for corporate social irresponsibility. Journal of Business Research, 66(10), 1822-1830.

Tabachnick, B. G., \& Fidell, L. S. (2013). Using multivariate statistics (6th ed.). Upper Saddle River, NJ: Pearson Education.

Trautwein, S., \& Lindenmeier, J. (2019). The effect of affective response to corporate social irresponsibility on consumer resistance behaviour: validation of a dual-channel model. Journal of Marketing Management, 35(3-4), 253-276.

Velasquez, M. G. (2012). Business ethics: concepts and cases (7th ed.). Upper Saddle River, NJ: Pearson Education.

Vignolli, E. (2016, 08 de abril). Empresários de Anchieta querem a volta da Samarco no ES: eles dizem que paralisação gerou desemprego e queda no faturamento. O Portal de Notícias da Globo. Recuperado de http://g1.globo.com/espirito-santo/ noticia/2016/04/empresarios-de-anchieta-queremvolta-da-samarco-no-es.html

Walker, K., Zhang, Z., \& Ni, N. (Nina). (2019). The mirror effect: corporate social responsibility, corporate social irresponsibility and firm performance in coordinated market economies and liberal market economies. British Journal of Management, 30(1), 151-168. 


\section{Mirian Albert Pires}

https://orcid.org/0000-0001-8975-9280

Doutora em Administração; Professora adjunta do Departamento de Ciências Contábeis da Universidade Federal do Espírito Santo (UFES). E-mail: mirian.pires@ufes.br

\section{Luciana Mourão}

https://orcid.org/0000-0002-8230-3763

Doutora em Psicologia; Professora titular do Programa de Pós-Graduação em Psicologia da Universidade Salgado de Oliveira (UNIVERSO). E-mail: mourao.luciana@gmail.com

\section{Fátima Bayma de Oliveira}

iD

https://orcid.org/0000-0001-5158-9546

Doutora em Educação; Professora Titular da Fundação Getulio Vargas na Escola Brasileira de Administração Pública e de Empresas (FGV EBAPE). E-mail: fatima.oliveira@fgv.br

\section{Jose Antonio Puppim de Oliveira}

https://orcid.org/0000-0001-5000-6265

Ph.D. em Planejamento (MIT); Professor da Fundação Getulio Vargas na Escola de Administração de Empresas de São Paulo (FGV EAESP) e na Escola Brasileira de Administração Pública e de Empresas (FGV EBAPE). E-mail: jose.puppim@fgv.br 